\title{
Zebrafish as a model to study autophagy and its role in skeletal development and disease
}

\author{
Joanna J. Moss ${ }^{1,2}$ (D) Chrissy L. Hammond ${ }^{2} \mathbb{D} \cdot$ Jon D. Lane $^{1} \mathbb{D}$ \\ Accepted: 31 August 2020 / Published online: 11 September 2020 \\ (c) The Author(s) 2020
}

\begin{abstract}
In the last twenty years, research using zebrafish as a model organism has increased immensely. With the many advantages that zebrafish offer such as high fecundity, optical transparency, ex vivo development, and genetic tractability, they are well suited to studying developmental processes and the effect of genetic mutations. More recently, zebrafish models have been used to study autophagy. This important protein degradation pathway is needed for cell and tissue homeostasis in a variety of contexts. Correspondingly, its dysregulation has been implicated in multiple diseases including skeletal disorders. In this review, we explore how zebrafish are being used to study autophagy in the context of skeletal development and disease, and the ways these areas are intersecting to help identify potential therapeutic targets for skeletal disorders.
\end{abstract}

Keywords Autophagy $\cdot$ Zebrafish $\cdot$ Bone $\cdot$ Development $\cdot$ Tools

\section{Introduction}

\section{Autophagy}

Autophagy is a catabolic process which enables the breakdown of cytosolic components into their basic biomolecular constituents by lysosomal degradation, so that they may be recycled for further use. It is an essential process required during cell differentiation and it contributes to the maintenance of cellular homeostasis where its primary function is to mobilise nutrients to sustain vital cellular functions during stress (Dikic and Elazar 2018). Since the first mechanistic descriptions of the autophagy process in 1967 by Christian de Duve (Deter and De Duve 1967), extensive research has been carried out to understand the autophagy pathways and their molecular control. Whilst these studies have established the importance of autophagy in cell differentiation

Chrissy L. Hammond

chrissy.hammond@bristol.ac.uk

Jon D. Lane

jon.lane@bristol.ac.uk

1 School of Biochemistry, Biomedical Sciences Building, University of Bristol, Bristol, UK

2 School of Physiology, Pharmacology and Neuroscience, Biomedical Sciences Building, University of Bristol, Bristol, UK and survival, they have also highlighted the vast number of housekeeping roles it plays, and the how its dysregulation contributes to the pathology of multiple diseases, including common skeletal disorders such as forms of arthritis and osteoporosis (Bouderlique et al. 2016; Cadwell and Debnath 2018; Jiang and Mizushima 2014; Levine and Kroemer 2019; Ochotny et al. 2013).

Autophagy can be divided into three main forms; chaperone-mediated autophagy (CMA), microautophagy and macroautophagy; each being delineated by the method of cargo delivery to the lysosome. This review will focus on macroautophagy (hereafter termed autophagy), as this considered to be the major form of autophagy and remains the most widely studied (Mizushima 2007). It involves the de novo formation of an intermediate organelle, the autophagosome, to deliver cargo to the lysosome for degradation. The autophagosome is a unique double-membrane structure which captures proteins, organelles, and other cellular debris before fusing with the lysosome to form a degradative autolysosome. This sequestration of cytosolic components can operate as a non-selective or selective process, with the former considered as a bulk, non-specific degradative process, whilst the latter requires specific receptor proteins to recognise and sequester target proteins, molecules, organelles, or invading pathogens. 


\section{Mechanism of autophagy}

The autophagy pathway is largely mediated by a family of highly conserved autophagy-related (ATG) proteins (Klionsky 2012; Wang et al. 2016), which were first discovered and characterised in budding yeast by Yoshinori Ohsumi and colleagues (Takeshige et al. 1992). To date, over 40 ATGs have been identified in yeast, most of which are conserved across higher eukaryotes (Kuma et al. 2017; Wei et al. 2018). Since their initial discovery, there has been an explosion of research focussed on delineating the fundamental mechanisms guiding the autophagic pathway. Broadly, this pathway can be split into three major steps: initiation and formation of the phagosome; phagosome elongation; and finally, lysosomal fusion. At each of these steps, dedicated ATG proteins and complexes are recruited and activated at distinct sites of phagophore assembly known as autophagosome initiation sites. As the nascent autophagosome is expanded, sealed, and trafficked to the lysosome, essential contributions are made by proteins coopted from other cellular membrane trafficking pathways such as ESCRT complex proteins, tethers and SNAREs (Lamb et al. 2013).

A comprehensive study of the full molecular machinery involved in the autophagy pathway is beyond the scope of this review (other in-depth reviews can be found here Boya et al. 2013; Dikic and Elazar 2018)). Instead, we will focus on the mammalian biology of core proteins thus far relevant to zebrafish autophagy models (Fig. 1). These include, the ULK1 complex, consisting of ULK1, ATG13, FIP200 and ATG101, and the phosphatidyl inositol 3-kinase complex I (PI3KC3), comprising VPS34, Beclin1 (BECN1), ATG14, AMBRA1 and p115, which are involved in autophagy initiation and phagophore formation. Next, the two conjugation systems: ATG5-ATG12ATG16L and MAP1LC3-ATG3 help cooperatively recruit and conjugate MAP1LC3 to the lipid phosphatidylethanolamine (PE) present on the phagophore membrane to form lipidated MAP1LC3-II. Prior to this step, ATG4 and ATG7 are responsible for processing MAP1LC3 into MAP1LC3-I ready for its lipid conjugation. These steps are central for autophagy detection and analysis, as fluorescently labelled MAP1LC3 - which appears as discrete $0.5-1.0 \mu \mathrm{m}$ puncta during autophagy - is the most commonly used marker for monitoring autophagy activity in cells and whole organisms. Finally, in the case of selective autophagy, receptor proteins such as p62/SQSTM-1, optineurin and NDP52 can specifically recognise and target polyubiquitinated cargo to the autophagosome.

Alongside these proteins, there are many other signalling pathways involved in initiating the autophagy response. Central to these are the mechanistic target of rapamycin complex 1 (mTORC1) pathway and the cAMP-dependent protein kinase A (PKA) pathway (Blommaart et al. 1995). It has become evident from a variety of studies in different

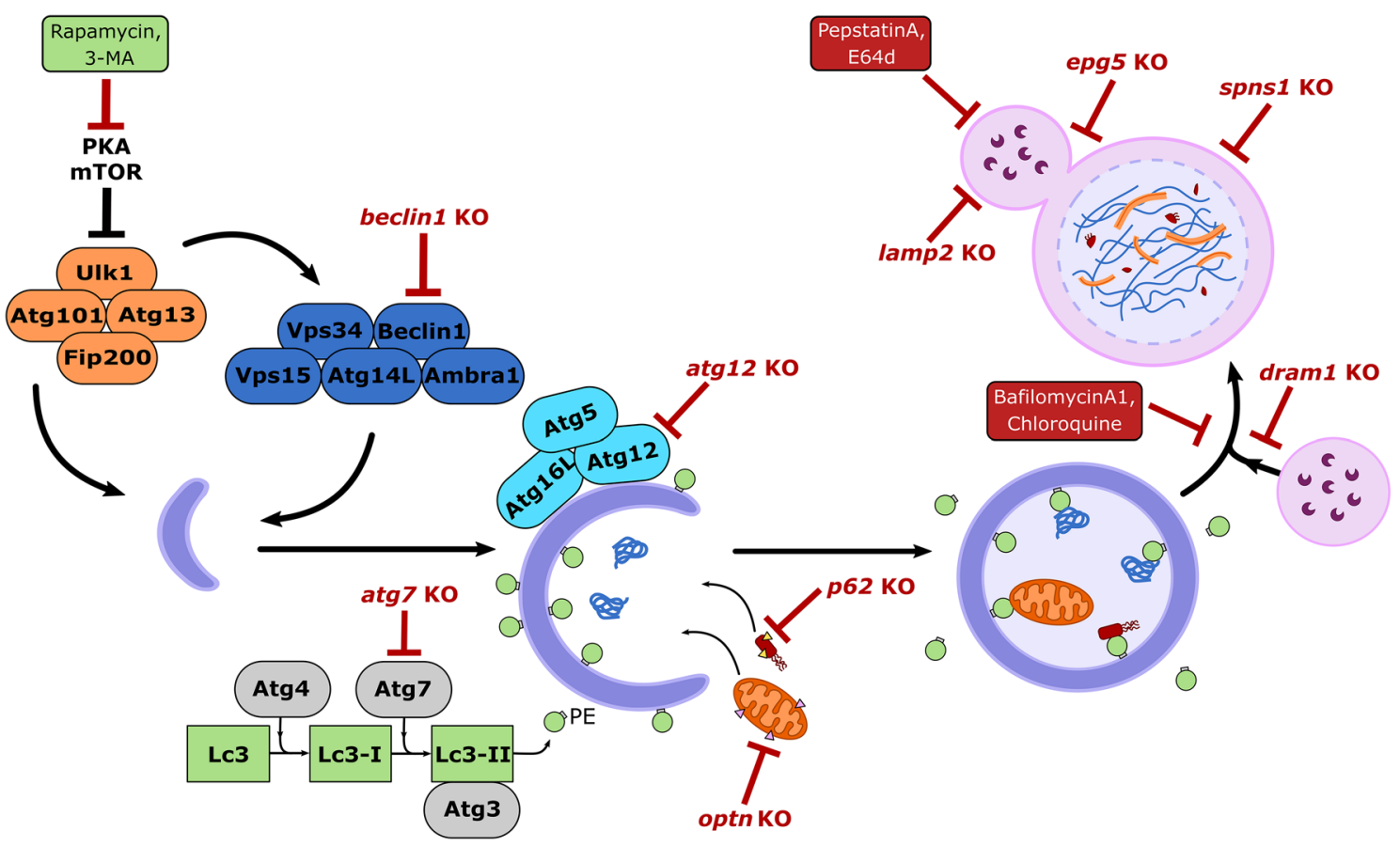

Fig. 1 Overview of the core proteins involved in the autophagy pathway and its regulation in zebrafish, knockout (KO) zebrafish lines highlighted in red, and boxes show commonly used drugs which can activate (green) or inhibit (red) autophagy activity. 
organisms that the proper regulation of autophagy initiation is essential for maintaining cellular homeostasis. Indeed, many studies have implicated autophagy dysregulation in the development of multiple neurological, cardiovascular, metabolic, and more recently, skeletal diseases (Choi et al. 2013; Wang et al. 2016; Wirawan et al. 2012). Meanwhile, other studies have uncovered a diversity of other cellular functions, beyond protein degradation, which are mediated by the autophagy machinery (Cadwell and Debnath 2018; Levine and Kroemer 2019). These include processes such as cellular differentiation and proliferation, cell metabolism, ER stress mitigation, and non-cell autonomous nutrient mobilisation, all of which are essential to bone and cartilage cell development and survival (Carames et al. 2010; Lee et al. 2015).

By understanding the distinct functions performed by autophagy in specialised cells and the key factors involved in its regulation, we can better grasp the influence of autophagy during the development of skeletal diseases, and how the autophagic pathway can be manipulated for therapeutic benefit. This review will focus on how zebrafish can be used to study autophagy in the context of skeletal development and the tools and techniques currently available within this area of research.

\section{Autophagy and skeletal development}

Multiple studies have implicated autophagy in the development and maintenance of the skeletal system. From the very early stages of development, autophagy plays essential roles in the differentiation, transformation and functional activity of key skeletal cells, such as osteoblasts (bone secreting cells), osteoclasts (bone absorbing cells), osteocytes (bone maintenance cells) and chondrocytes (cartilage secreting cells) (Fig. 2) (Aghajanian and Mohan 2018). Post-development, emerging evidence shows that once terminally differentiated, these cells require a constitutive level of basal autophagy to ensure proper functioning and survival in the hypoxic, nutrition-deficient and hypertonic environments they reside in (Mizushima and Levine 2010).

During embryonic development, the vertebrate skeleton, and its associated bone, cartilage, and connective tissues, are formed from mesenchymal stem cells (MSCs). These cells are originally derived from three distinct embryonic cell lineages which go on to establish different regions of the skeleton (Hall and Miyake 1992). To form the skeleton, cells from these lineages first migrate to the appropriate region within the embryo for skeletal formation, where they aggregate and proliferate to form mesenchymal condensations that subsequently differentiate into either osteoblasts or chondrocytes. Studies have highlighted vital roles for autophagy within this differentiation process and within the differentiation capabilities of MSCs. For example, MSCs have been shown to have high levels of basal autophagy (Oliver et al. 2012), whilst treatment of MSCs isolated from young mice with the class III PI3Kinase inhibitor 3-methyladenine (3-MA), which blocks autophagy induction, was shown to cause a reduction in the capacity of MSCs to differentiate into osteoblasts (Ma et al. 2018). Further to this, a study in primary human MSCs showed that during osteoblast differentiation from
Fig. 2 Overview of the roles autophagy plays in bone and cartilage cells, autophagy helps maintain the homeostasis, survival and function of osteoblasts, osteoclasts, osteocytes and chondrocytes

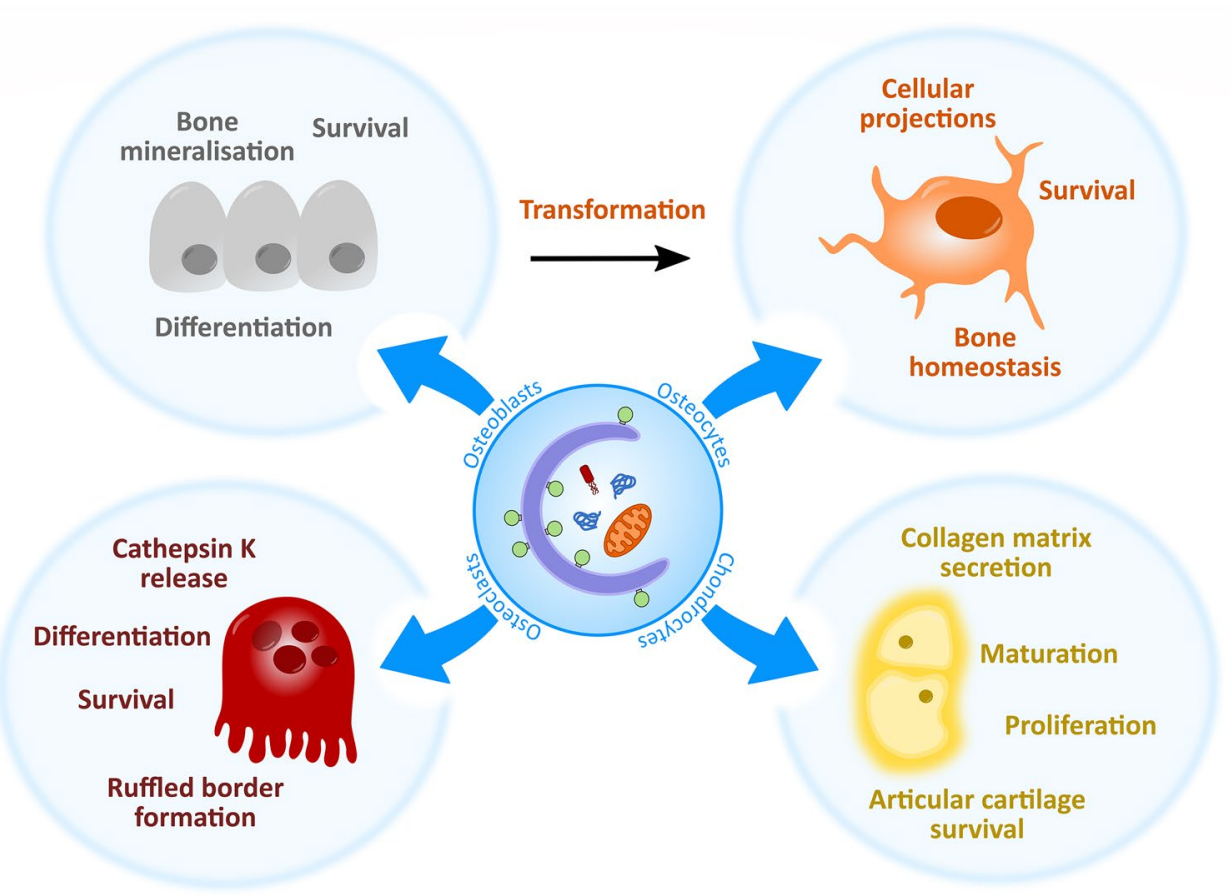


MSCs, these cells accumulate large numbers of autophagic vacuoles which are later broken down to provide energy (Nollet et al. 2014). The authors concluded that autophagy is required to help balance energy supply during the differentiation process and that it is therefore vital for MSC differentiation and function (Nuschke et al. 2014). An in vitro study using primary murine osteoblast cells also showed that blocking autophagy through the knockout of FIP200, an essential component of the mammalian ULK1 complex, inhibited osteoblast differentiation, further demonstrating the importance of autophagy during the establishment of the osteoblast population (Liu et al. 2013).

From osteoblast and chondrocyte cells, bone is formed via two different mechanisms. In intramembranous ossification, bone is secreted directly by osteoblasts which is how much of the craniofacial skeleton, vertebral column, and fins are formed in zebrafish (Bird and Mabee 2003). Conversely, in endochondral ossification, chondrocytes first form a cartilaginous skeletal template which is then gradually replaced by bone. For tetrapods, this is how long bones form, whilst in zebrafish the ceratohyal and hyperurals are formed this way (Mundlos and Olsen 1997). As the organism develops, bone is lengthened and modelled until the final skeleton is formed, although constant remodelling of the skeleton in response to changes in mechanical loading or bone fractures continues throughout life.

This process of bone modelling and remodelling is mediated by osteoblasts, osteoclasts, and osteocytes. Osteoblasts line the surface of the bone and are responsible for synthesising, secreting, and mineralising the bone matrix. Osteoblasts that become trapped within bone further differentiate into osteocytes which interact as a mechano-sensing network and direct the recruitment of osteoblasts and osteoclasts to local bone areas. Meanwhile, osteoclasts differentiate from hematopoietic precursors and migrate to areas of active bone remodelling to help degrade and resorb bone. The coordinated activity of these cells is essential to ensuring bone homeostasis as disturbances to this equilibrium can lead to disease (Table 1). Autophagy is one process that has been shown to be essential for maintaining this balance and for regulating bone and cartilage cell differentiation, formation, and function.

\section{Autophagy in bone formation}

Osteoblasts are the primary cell type responsible for bone formation and both their survival and function are regulated by autophagy. The differentiation of osteoblasts from MSCs is regulated by the transcription factors RUNX2 and SP7 (also known as Osterix). During this process, studies have shown that autophagy is upregulated to help these cells survive the hypoxic bone environment and to combat oxidative stress (Nollet et al. 2014), as manipulation of autophagic activity levels positively correlates with osteoblast survival (Li et al. 2017).

Beyond survival, autophagy activity is closely linked to osteoblast mineralisation. Osteoblasts form mineralised bone through the deposition of hydroxyapatite crystals into the collagen based bone matrix. As the matrix matures, these crystals are organised into a lattice structure within the collagen, forming bone. These hydroxyapatite crystals have been identified in autophagic vacuoles within osteoblasts, and the inhibition of autophagic flux has been shown to block the outward extrusion of these minerals (Nollet et al. 2014). Further to this, depletion or deletion of ATG5, ATG7 or Beclin1, which are essential for autophagosome formation, have all been shown to cause decreased bone mass and mineralisation (Nollet et al. 2014; Zahm et al. 2011). In the case of targeted ATG7 loss in mice, an increased number of bone fractures were recorded and suggested to be associated with induced ER stress and decreased osteoblast numbers (Li et al. 2018; Piemontese et al. 2016). Meanwhile, another in vitro study showed that deletion of FIP200 in osteoblasts impaired their terminal differentiation, inhibiting bone formation, and causing an osteopenia phenotype (Liu et al. 2013).

There are several bone disorders linked to osteoblast dysfunction whose pathogenesis has also been linked to autophagy dysregulation, such as Osteogenesis Imperfecta and osteoporosis (Table 1). For all bone disorders which are caused by excessive and disorganised or insufficient bone formation, treatment options are limited. This is because there are few drugs available which can effectively and safely target and promote osteoblast numbers and activity (Kawai et al. 2011; Riggs and Parfitt 2005). Therefore, influencing osteoblast activity through an alternative target, such as autophagy, could be a useful therapeutic mechanism, emphasising the need for more research into understanding the role of autophagy in osteoblast development and function.

\section{Autophagy in bone resorption}

At sites of bone remodelling, hematopoietic mononuclear myeloid stem cells (HSCs), primarily residing in the bone marrow, can differentiate into osteoclasts and migrate to the bony tissue surface in a process mediated by colonystimulating factor 1 (CSF-1) and RANK ligand (RANKL) (Zhao et al. 2007). Once differentiated and activated, osteoclasts attach to the bone surface, forming a seal via actin rings and begin bone resorption through the secretion of lysosomal proteases, metalloproteinases, and cathepsin $\mathrm{K}$ (CTSK). In mice, autophagy has been shown to play a role in osteoclast differentiation as HSC-specific loss of ATG7 caused increased genomic and cellular damage to HSCs and a failure to differentiate (Mortensen et al. 2011), indicating that autophagy may be important for the maintenance of 


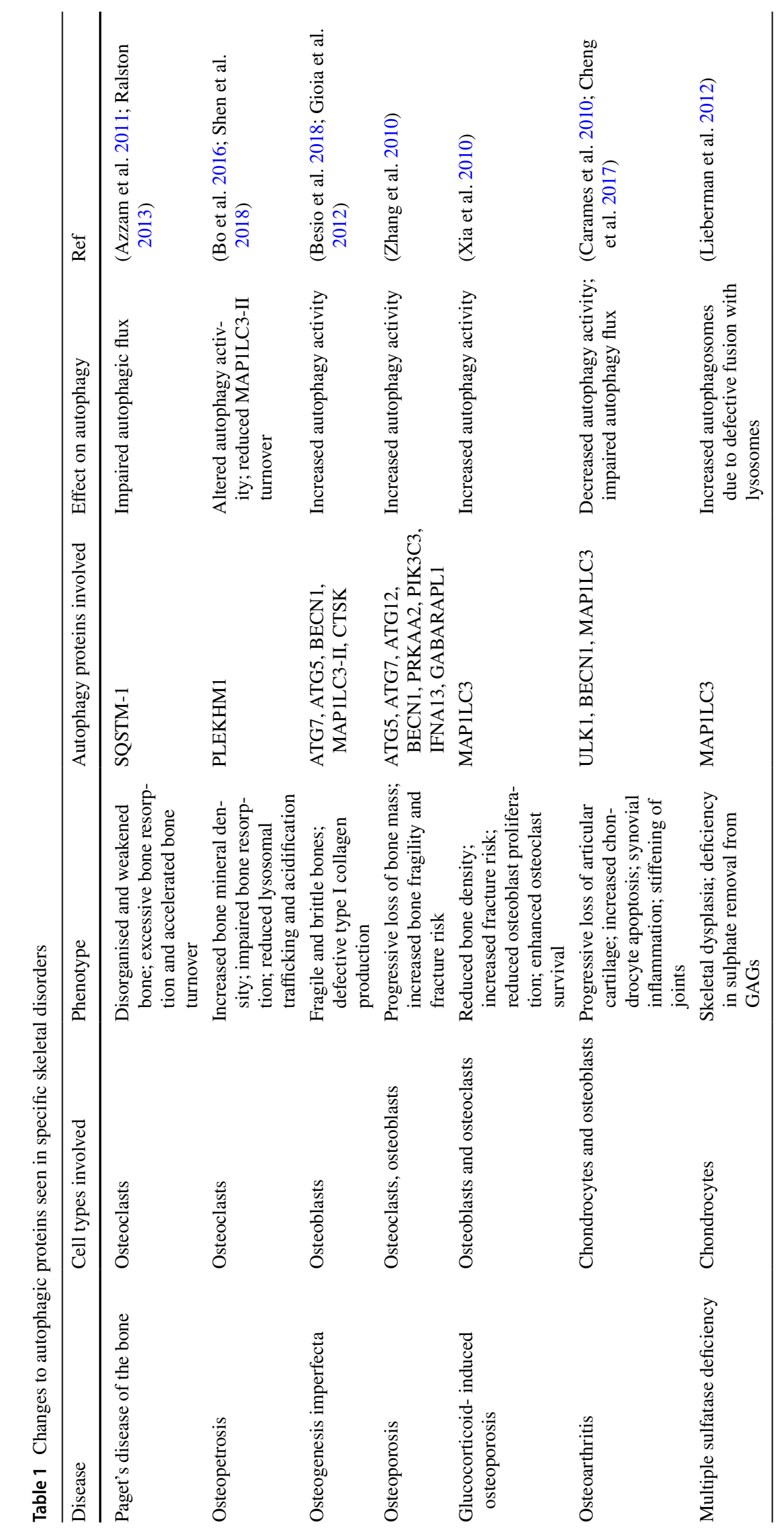


HSCs. In addition to differentiation, autophagy helps osteoclasts survive in the locally hypoxic environment of the bone surface. In vitro studies have shown that in hypoxic environments, autophagy is upregulated in osteoclasts to reduce cell stress and to protect against apoptosis (Wang et al. 2011; Zhao et al. 2012). Correspondingly, increased autophagy activity enhances osteoclast differentiation (Shi et al. 2015).

Autophagy has also been shown to be involved in osteoclast activity and function. Osteoclasts resorb bone through the secretion of matrix-degrading molecules onto bone via secretory lysosomal vesicles. They have a characteristic ruffled border where the exocytosis of lysosomes occurs, and these lysosomes have been shown to be labelled with MAP1LC3 (DeSelm et al. 2011). Through the use of conditional knockout mouse models, roles for the ATG conjugation machinery in osteoclast formation and resorptive activity have been suggested (DeSelm et al. 2011). Loss of ATG5 and ATG7 has been shown to impair ruffled border formation and lysosomal trafficking and secretion, causing a reduction in bone-resorption capacity, and increasing trabecular bone volume. These mice also showed a decrease in MAP1LC3 and RAB7 (a RAB GTPase) localisation to the ruffled border and inhibited CTSK release, which likely explains the effect on resorptive activity. Similarly, MAP1LC3A - a specific sub-form of MAP1LC3 - was knocked down, both actin ring formation and CTSK release were blocked, thereby inhibiting resorption activity (Chung et al. 2012). Taken together, these data suggest that ATG proteins have a clear role in regulating osteoclast activity. This is further highlighted in the pathogenesis of bone disorders caused by defective osteoclast function which are also linked to mutations in autophagy related proteins, as mentioned in Table 1.

\section{Autophagy in bone maintenance}

Osteocytes are terminally differentiated cells formed from osteoblasts which have become trapped within the bone matrix. They are vital for bone health and maintenance and are responsible for regulating the bone remodelling process. Through the extension of dendrite-like processes within the bone matrix, osteocytes connect to form a vast network which detects and responds to hormonal and mechanistic changes within the bone environment by directing the recruitment of osteoblasts and osteoclasts to local bone areas.

Just as autophagy has a key role in osteoblast differentiation and function, autophagy has been shown to be important for osteocyte health and maintenance. Firstly, during the osteoblast to osteocyte transition, the cells must undergo a dramatic change in cell shape and composition which requires an active recycling of organelles (Dallas and Bonewald 2010). Secondly, a study using human and rat bone tissue demonstrated that osteocytes show an accumulation of MAP1LC3 puncta and that this expression is higher in osteocytes than osteoblasts (Zahm et al. 2011). This indicates a high basal level of autophagy which is likely to be necessary to survive the nutrient and oxygen poor environment of the bone matrix.

Looking into the role of autophagy in osteocyte functioning, when autophagy activity was inhibited in mice by the osteocyte specific deletion of ATG7, there was a significant decrease in bone mass which was associated with reduced osteoblast and osteoclast numbers, and a disturbance in bone homeostasis (Onal et al. 2013). Similarly, in mice with ATG7 deficient osteoblasts, osteocytes showed decreased cellular projections and reduced ER degradation and turnover (Piemontese et al. 2016). Together, these results indicate a clear role for autophagy in osteocyte function, whilst also demonstrating the level of interaction between these skeletal cell types and how the dysfunction of one can impact the activity of others. This, therefore, has a significant impact upon bone health and homeostasis and should be an important consideration when studying bone disorders and selecting possible drug targets.

\section{Autophagy in cartilage formation and maintenance}

Alongside bone cells, cartilage forming chondrocytes also play a critical role within skeletal development and function. Chondrocytes are responsible for forming both the initial cartilaginous skeleton during endochondral ossification and the articular cartilage layer between bones, which enables fluid joint movement. As with the other skeletal cell populations, autophagy has been shown to be a vital process for chondrocyte differentiation, function and survival (Vuppalapati et al. 2015; Zhang et al. 2013).

During endochondral bone formation, chondrocytes form the cartilage anlage of the future bone through the secretion of a collagen-rich matrix. This process continues until the chondrocytes reach a non-proliferative, hypertrophic state at which point the cells undergo apoptosis, triggering the resorption of cartilage and its mineralisation into bone by invading osteoblasts (Berendsen and Olsen 2015). Some chondrocytes remain within regions near to the end of the forming bone known as growth plates, where the chondrocytes continue to proliferate and secrete a cartilage matrix to enable longitudinal bone growth via ossification. During this process of chondrocyte proliferation and differentiation, in vitro studies have shown there is a positive correlation with levels of autophagy activity (Vuppalapati et al. 2015), and maturing chondrocytes show high MAP1LC3 expression (Srinivas et al. 2009). In growth plate chondrocytes, mice with a chondrocyte specific deletion of ATG7 showed impaired matrix secretion due to the retention of synthesised type II procollagen (a major component of cartilage matrix) 
within the ER (Cinque et al. 2015). Meanwhile another study showed that the conditional loss of ATG5 or ATG7 in mice enhanced chondrocyte cell death and decreased cell proliferation resulting in reduced growth plate activity and growth retardation (Vuppalapati et al. 2015).

Joint articular cartilage is retained throughout life, although due to the limited regenerative and repair capabilities of articular cartilage, homeostatic mechanisms such as autophagy are vital for its maintenance and preservation (Barranco 2015; Zhang et al. 2013). For example, healthy human cartilage shows a high expression of key autophagy factors such as ULK1, Beclin1, and autophagosome-associated MAP1LC3-II, indicating that autophagy is a constitutively active mechanism within cartilage (Caramés et al. 2010). Meanwhile, mice with a conditional knockout of ATG5 in chondrocytes showed increased articular chondrocyte cell death, which escalated with age, and an accumulation of p62 in the articular cartilage, indicating abrogated autophagic flux (Bouderlique et al. 2016). By 1 year of age, these mice had experienced significant or complete loss of articular cartilage at joint sites and severe development of the joint disease osteoarthritis (OA). Together, these results suggest that autophagy may play a protective role against cartilage degradation by maintaining chondrocyte health and survival. This is further supported by data demonstrating that patients with osteoarthritis, which is characterised by the progressive loss of articular cartilage, show decreased expression of key autophagy markers which continue to decline further as the severity of the disease increases (Carames et al. 2010).

\section{Zebrafish as a model to study autophagy during skeletal development and pathology}

As discussed above, it is clear that autophagy is an important player within bone and cartilage cell development and maintenance, and that its activity is vital for sustaining skeletal homeostasis. Indeed, there are multiple skeletal disorders that are triggered by an imbalance in bone or cartilage cell activity with accompanying dysregulated autophagy activity. Many of these disorders are chronic and debilitating, and currently have limited treatment options available. Therefore, expanding our understanding of the cellular and molecular processes that are central to the co-ordination of bone and joint development, such as autophagy, will be crucial for the advancement of new therapeutics for these diseases, as well as for broadening our understanding of their pathogenesis.

Much has been learnt about the skeletal system and its associated disorders through the use of animal models (Gomes and Fernandes 2011). In vivo models offer obvious advantages over in vitro cell models for skeletal research as the complex, moveable, three-dimensional structure of bones and joints cannot be fully recapitulated within an in vitro system. Equally, the effects of other cell and tissues types and their related secretions on cartilage and bone cells cannot be recaptured within a unicellular system. Whilst a number of different animals have been used as models for bone research, mouse models remain the most extensively used due to their generally lower husbandry cost, fast generation times, ease of handling and genetic tractability (Sommer et al. 2019). However, despite these advantages, rodent models do have some inherent limitations for bone and autophagy-based research such as, differential bone loading compared to humans, and a lack of visual accessibility at a cellular level and during early developmental stages.

Increasingly, zebrafish (Danio rerio) are being recognised as a useful alternative to rodent models for skeletal and autophagy research. Firstly, zebrafish are highly fecund, with a single pair able to lay up to 300 eggs a week, which develop externally as optically translucent larvae. This allows for the study of both cellular and gross morphological changes during early development, without the need for invasive experimental techniques or animal sacrifice. Secondly, zebrafish are highly genetically tractable, as through the use of genetic tools such as TALEN (Bedell et al. 2012) and CRISPR/Cas9 (Talbot and Amacher 2014) embryos can be injected with constructs at the single cell stage to generate transgenic or genetically altered zebrafish lines. As these tools continue to improve, and with access to a fully sequenced genome, it is possible in zebrafish to efficiently and specifically target multiple genes in a high-throughput manner (Liu et al. 2019). It is through such methods that many knockout and reporter lines have been developed and used to model specific diseases or to visualise and track specific proteins or cell types, such as bone cells or autophagy related proteins.

As vertebrates, zebrafish show a high genetic similarity to humans (Kabashi et al. 2011), and all of the core mammalian autophagy-related proteins can be found within the zebrafish genome, with the overall amino acid identity between these and their human counterparts ranging between 40 and $96 \%$ (Mathai et al. 2017). This high degree of conservation indicates that the autophagy pathway operates in a very similar way within zebrafish compared to humans and has enabled the development of many mutant and transgenic autophagy zebrafish lines (Table 2).

Despite clear structural differences, zebrafish also share similar skeletal physiology to mammals, including the same joint types and joint components such as joint cavities, articular cartilage and synovial membranes (Askary et al. 2016). This has been most widely shown in the larval zebrafish jaw which has been extensively studied and remains one of the main joint sites used to model joint development. Additionally, the overall molecular mechanisms underlining 


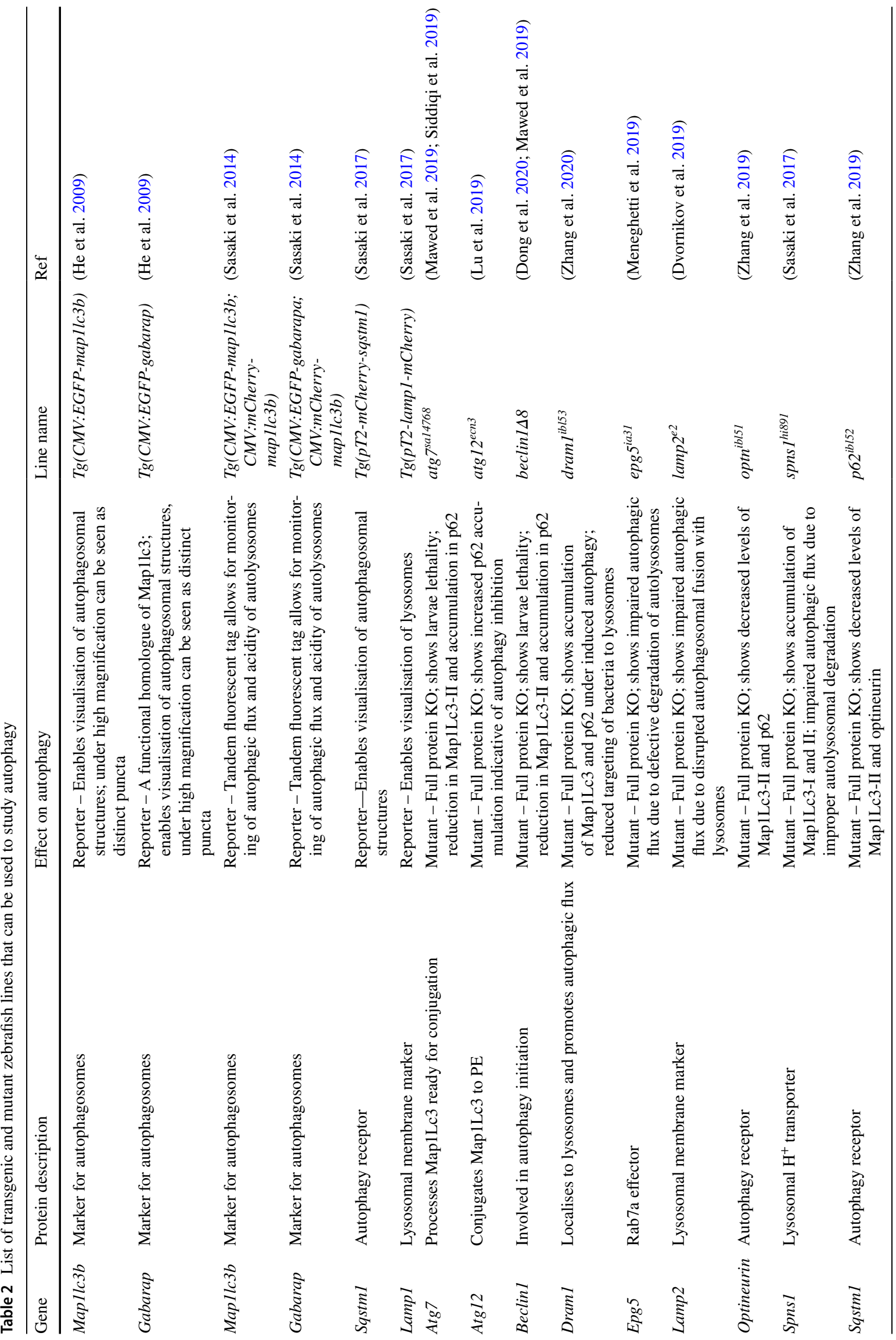


vertebrate skeletal segmentation, joint development, and fin/ limb development are very similar and are likely to have been conserved across species (Crotwell and Mabee 2007).

Many human skeletal disorders can be modelled in zebrafish and can recapitulate the phenotypes seen in higher vertebrates. For example, equivalent models for disorders such as osteogenesis imperfecta, scoliosis, osteoporosis, Stickler syndrome, and osteoarthritis are available in zebrafish (Askary et al. 2016; Carnovali et al. 2019; Lawrence et al. 2018; Mackay et al. 2013). Additionally, as zebrafish develop osteoarthritis naturally during ageing, the pathogenesis of the disease and its common symptoms such as increased spinal deformities, vertebral dislocations, and fractures, and the formation of osteophytes can be easily explored (Hayes et al. 2013). Taken together, these data demonstrate that zebrafish are representative, relevant, and useful models for the study of bone and joint development, the pathology of skeletal disorders, and the genes involved in either processes. They also present some unique advantages for the dynamic and real-time study of autophagy activity and expression within different skeletal cell types and systems from early development to adulthood.

\section{Current tools available to study autophagy in zebrafish}

\section{Transgenic and mutant zebrafish lines}

Given the genetic and physiological similarities between humans and zebrafish, multiple transgenic and mutant lines targeting key autophagy and skeletal genes have been developed in zebrafish (for common skeletal zebrafish lines, see (Bergen et al. 2019)). Although these lines have been well used for studies within their respective fields, much less research has been done using autophagy and skeletal zebrafish lines in combination.

The first transgenic autophagy reporters generated in zebrafish were the GFP-Map1Lc3 and GFP-Gabarap transgenic lines, with reporters expressed under the control of the constitutive cytomegalovirus (CMV) promoter (He et al. 2009) (Table 2). Both Map1Lc3 and Gabarap are homologues of yeast Atg8, and each form a subfamily of proteins in mammals and fish. In mammalian cells, the MAP1LC3 and GABARAP family members act cooperatively to enable autophagosome formation and/or cargo recognition, and as such, are equally useful for measuring autophagy in vivo and in vitro. However, overall, Map1Lc3 is the most widely used marker for identifying and visualising autophagy activity. During autophagy, GFP-Map1Lc3 acts like endogenous Map1Lc3 and becomes conjugated to PE in the developing phagophore and remains associated with the autophagosome until its full closure (Fig. 1). Using fluorescence microscopy, this lipidated form of GFP-Map1Lc3-II can be visualised as puncta or as ring-like structures as shown in Fig. 3 (Kabeya et al. 2000; Mizushima et al. 2004).

The GFP-Map1Lc3 transgenic zebrafish line has been used in several studies to explore the role of autophagy in bacterial clearance (Mostowy et al. 2013), blastema formation following fin amputation (Varga et al. 2014), and in liver homeostasis (Cui et al. 2012). With regards to skeletal biology, this line can also be used to explore the expression pattern of autophagy during early development and to identify cell types showing high expression of autophagy activity. As shown in Fig. 3, we were able to identify that during development, zebrafish show high expression of GFP-Map1Lc3 around joint sites, and that the cells in the joint interzone specifically show increased GFP-Map1Lc3 expression compared to the surrounding cells. Given the optical clarity of zebrafish, these fish can be imaged live under anaesthetic, and the expression of Map1lc3 can be tracked throughout development in the same fish.

Moreover, through the use of skeletal cell specific transgenic lines, the expression of GFP-Map1Lc3 can be correlated to a specific cell type. For example, transgenic lines expressing Col10a1 or sp7 can be used to label osteoblasts at different stages of differentiation; transgenic GFP-trap fish can be used to label osteoclasts; whilst fluorescent labelling of type II collagen can be used to monitor chondrocytes and cartilage development and formation. Similarly, there are many antibodies and transgenics available for the labelling of muscles, collagens, tendons, and ligaments, enabling the expression of Map1Lc3 to be explored in many different cell types related to the skeletal system. This can allow for the identification of patterns in autophagy activity during skeletal patterning and differentiation, either in normal development, in response to the mutation of genes of interest, or during pharmacological (Seda et al. 2019) or mechanical manipulation (Brunt et al. 2016). These data could be correlated to phenotypes seen in disease models to explore how autophagy activity may be being altered within different bone disorders. There are also several autophagyspecific knockout zebrafish lines which have been shown to block or impair autophagy activity (Table 2). These enable investigations of how loss of autophagy activity affects the differentiation and function of skeletal cells and the gross development and performance of the skeleton.

While expression of GFP-Map1Lc3 can be very useful for identifying where autophagy may be upregulated, its expression alone cannot be used to determine autophagic flux or dynamics. For example, an increase in Map1Lc3 puncta could be due to elevated autophagy activity, and/or due to impaired lysosomal clearance (i.e. impaired autophagic flux) (Klionsky et al. 2016). To measure autophagic flux in zebrafish, lysosomes can be labelled with either a dye such as LysoTracker (Fig. 3b), or a fluorescent probe, such 

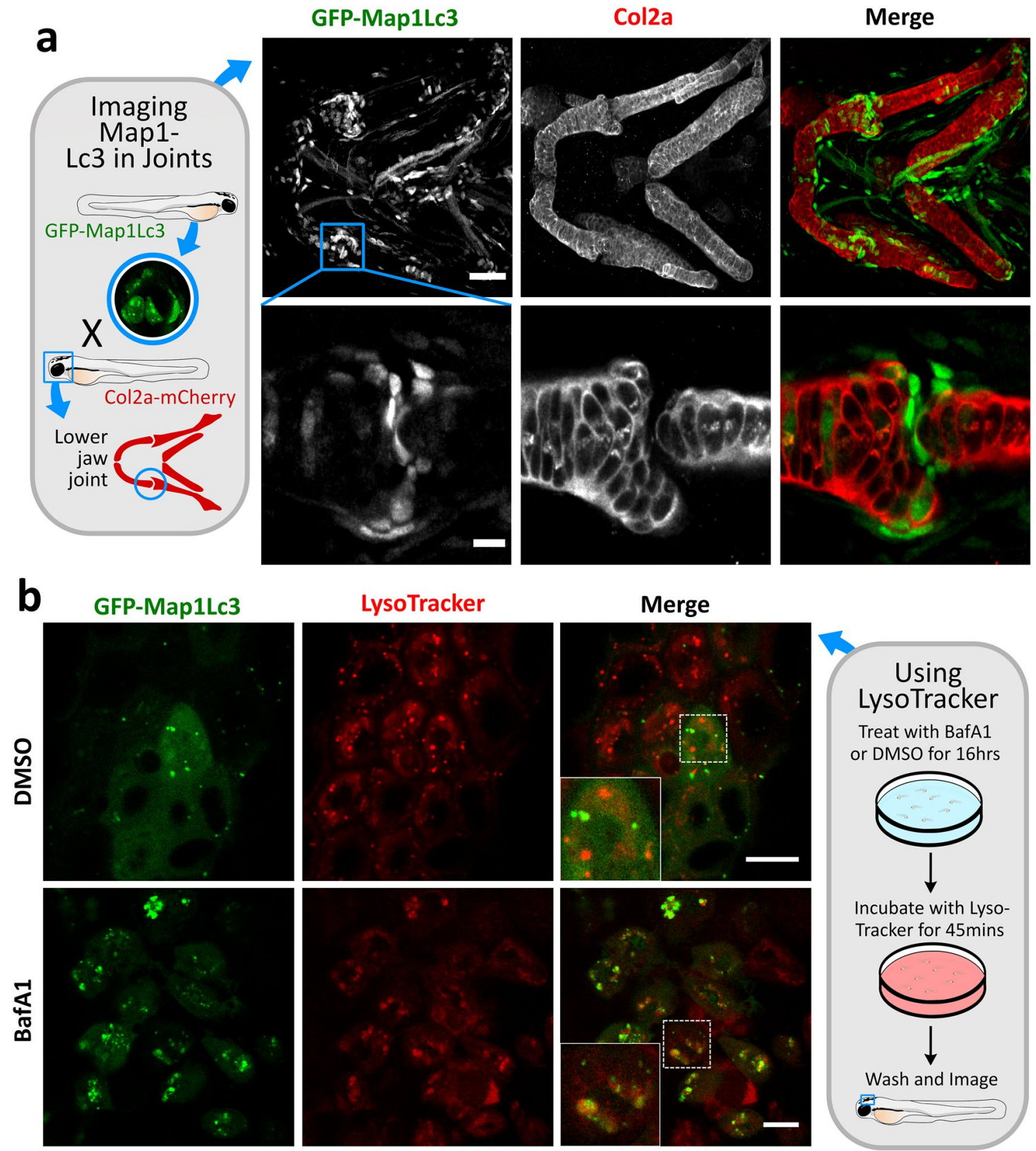

Treat with BafA1 or DMSO for $16 \mathrm{hrs}$

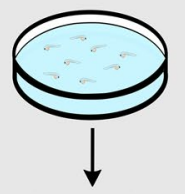

Incubate with LysoTracker for $45 \mathrm{mins}$

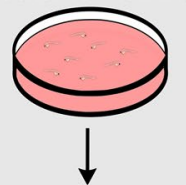

Wash and Image

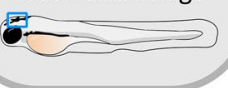

Fig. 3 Examples of how GFP-Map1Lc3 transgenic zebrafish line can be used to study autophagy in a skeletal context, a Confocal images of the lower jaw and lower jaw joint of a transgenic CMV:EGFPmapllc $3 b$ zebrafish at 3 days post fertilisation (dpf), immunostained for collagen Type II (red) and anti-GFP for lc3 (green). Scale

as mCherry-Lamp1 or Lamp2 (Sasaki et al. 2017). In this way, colocalisation between GFP-Map1Lc3 and a lysosomal marker can be used to assess the level of autophagic activity. Similarly, drugs which block autolysosome formation (e.g. lysosomal $\mathrm{H}^{+}$-ATPase inhibitors), and therefore prevent the turnover of Map1Lc3 puncta, can also be applied to assess autophagic flux (Fig. 1). However, when using autophagy modulators it is important to consider their full bar $=50 \mu \mathrm{m}$ and $10 \mu \mathrm{m}$, respectively. b Confocal images of epidermal cells in transgenic $C M V: E G F P$-mapllc $3 b$ zebrafish imaged at $5 \mathrm{dpf}$ after treated with $100 \mu \mathrm{l}$ BafilomycinA1 or DMSO for $16 \mathrm{~h}$ followed by $45 \mathrm{~min}$ live staining in red LysoTracker dye. Inset boxes show zoom of white checked box. Scale bar $=10 \mu \mathrm{m}$

effects in vivo, as several target other non-autophagic pathways such as the endocytic pathway, or cause other indirect effects (Klionsky et al. 2016).

Dual fluorescent probes, or tandem-tags, such as mCherry-GFP-Map1Lc3, are also very useful for analysing autophagy flux. Unlike the fluorescent GFP probe, mCherry is less sensitive to the acidic environment of the lysosome, and therefore is not quenched. By measuring the proportion 
of yellow (green and red puncta together) and red puncta (autophagosomes and autolysosomes, respectively), the level of autophagic flux activity can be estimated. Together, these tools provide a more accurate way to measure autophagy activity and differences in autophagy levels quantitatively between different fish. Indeed, these tools have been used in zebrafish studies to determine how autophagic flux is affected by specific genetic mutations within bone and cartilage cells (Hu et al. 2019; Santos-Ledo et al. 2017). These studies showed that changes to bone and cartilage cell differentiation and functioning were in part due to dysregulated autophagy activity. Therefore, these tools could also be used to help establish whether bone disorders alter autophagic flux within specific cells and to explore the potential benefit of autophagy-modulating drugs at addressing this imbalance.

Recently, there has been increased interest in using zebrafish scales as model for imaging bone cell dynamics (Bergen et al. 2019; Pasqualetti et al. 2012). Although their structure is simpler compared to mammalian bones, several studies have shown that osteoclasts and osteoblasts can respond to hormones and other substances indicating that their activity and fundamental regulation is unchanged (Carnovali et al. 2016; de Vrieze et al. 2015; Park et al. 2016). Therefore, zebrafish scales offer a new in vivo bone model for the live imaging of osteoclasts and osteoblasts in fluorescent transgenic lines. Looking ahead, this model could be used to study autophagy dynamics in bone cells using transgenic autophagy lines, or to screen autophagy modulating drugs for their effects on bone cell activity.

\section{High-throughput drug screening}

Over the last 20 years, zebrafish larvae have become an established model for high throughput chemical screens (Cully 2019; Rennekamp and Peterson 2015). Utilising the transparency, small size, and ease of drug administration, zebrafish can provide both the high-throughput capabilities of an in vitro system with the full complexities of whole organism biology. Combining this system with high-resolution imaging and fluorescent reporters/dyes means that the effects of putative drugs on a specific cellular pathway or disease mutation can be rapidly quantified, assessed and validated (Early et al. 2018; Mathias et al. 2012; Walker et al. 2012). To date, eleven compounds have been identified through zebrafish screens as potential therapeutics, of which nine are in or about to enter early clinical trials (Cully 2019), including one for the connective tissue disorder, Fibrodysplasia ossificans progressive. As several skeletal disorders can be accurately modelled in zebrafish, this system could be applied as a primary screening platform for identifying and testing potential therapeutics for these disorders. Using autophagy reporter lines, it could also be used to identify new autophagy modulating drugs or to test the efficacy of current modulators on skeletal disease pathology. For example, the GFP-Map1Lc3 line has been used to validate the effect of potential autophagy enhancers, such as AUTEN67, identified through small-molecule library screens (Papp et al. 2016). Meanwhile, Khuansuwan et al. used a neuronspecific GFP-Map1Lc3b line to validate the effect of two, in-trial drugs for Parkinson's as autophagy modulators (Khuansuwan et al. 2019).

As mentioned previously, interest is growing in the use of zebrafish scales as an ex vivo model. This model has the advantage that screens can be performed on scales harvested from a single fish thereby reducing intra-individual variation, and it allows for compounds to be tested in the context of homeostasis within a mature tissue (de Vrieze et al. 2015). Already, this model has been used to study the effect of different drugs on bone cells (Park et al. 2016), and to screen compound libraries to identify new osteo-anabolic and catabolic drugs (de Vrieze et al. 2015). Similarly, this system has been proposed for the identification of drugs for osteoporosis, whereby scales collected from fish with osteoporosis-specific mutations are used to screen for possible therapeutic drugs (Bergen et al. 2019). Together, both of these systems show enormous potential as primary testing platforms and demonstrate how zebrafish can be used to provide fast, functional validation of prospective new drugs.

\section{Bone repair and regeneration assays}

As zebrafish fins and scales remain optically accessible throughout adulthood, zebrafish provide a useful tool for dynamically observing the factors involved in bone regeneration and bone repair (Fig. 4). Similar to other teleosts, zebrafish can regenerate parts of their body following amputation. In the fins, this regeneration process is relatively quick and within two weeks all major tissue, including bone, joints, and nerves are largely restored (Watson and Kwon 2015). Using a fin regeneration assay, Varga et al. explored the role of autophagy within this process and showed that the genetic and pharmacological inhibition of autophagy impairs fin regeneration (Varga et al. 2014). This highlighted an important role for autophagy in tissue patterning and renewal. Further investigations into this process could be helpful for identifying how manipulation of autophagy can be used to promote bone and cartilage cell renewal and replacement, especially during ageing.

Zebrafish are also a useful model for fracture repair studies as they show a fracture healing response with callus formation which is very similar to mammals (Sousa et al. 2012; Tomecka et al. 2019). Using the GFP-Map1Lc3 line and the live bone stain, Alizarin red, we were able to perform a fin fracture assay which showed that the expression of Map1Lc3 increases during the early stages of fracture repair (Fig. 4). This indicates that the autophagy activity may be 
Fig. 4 Using GFP-Map1Lc3 transgenic zebrafish line to study the role of autophagy in fin fracture repair and bone regeneration - Top, Schematics depicting how bone fracture repair and regeneration assays can be performed in zebrafish and how live staining of bone can be performed in adult zebrafish using Alizarin Red. Bottom, Fluorescent stereomicroscope images of a fracture repair assay performed in a transgenic $C M V: E G F P$ mapllc $3 b$ zebrafish at 6 months post fertilisation, live stained with Alizarin Red (red). Dpi days post injury; scale $\mathrm{bar}=200 \mu \mathrm{m}$. Figure made in collaboration with Miss Lucy McGowan

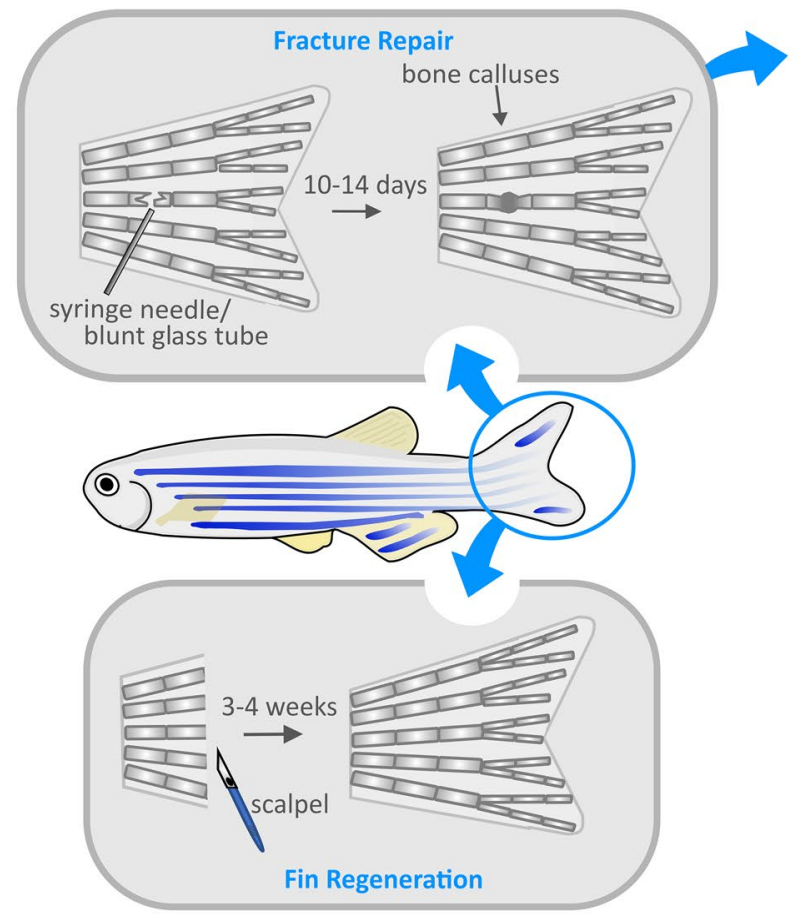

Stain bone with Alizarin

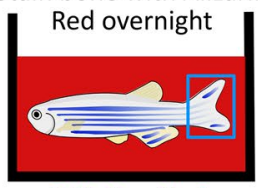

GFP-Map1Lc3
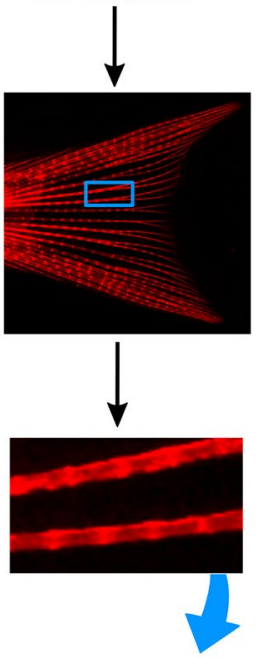

Alizarin Red
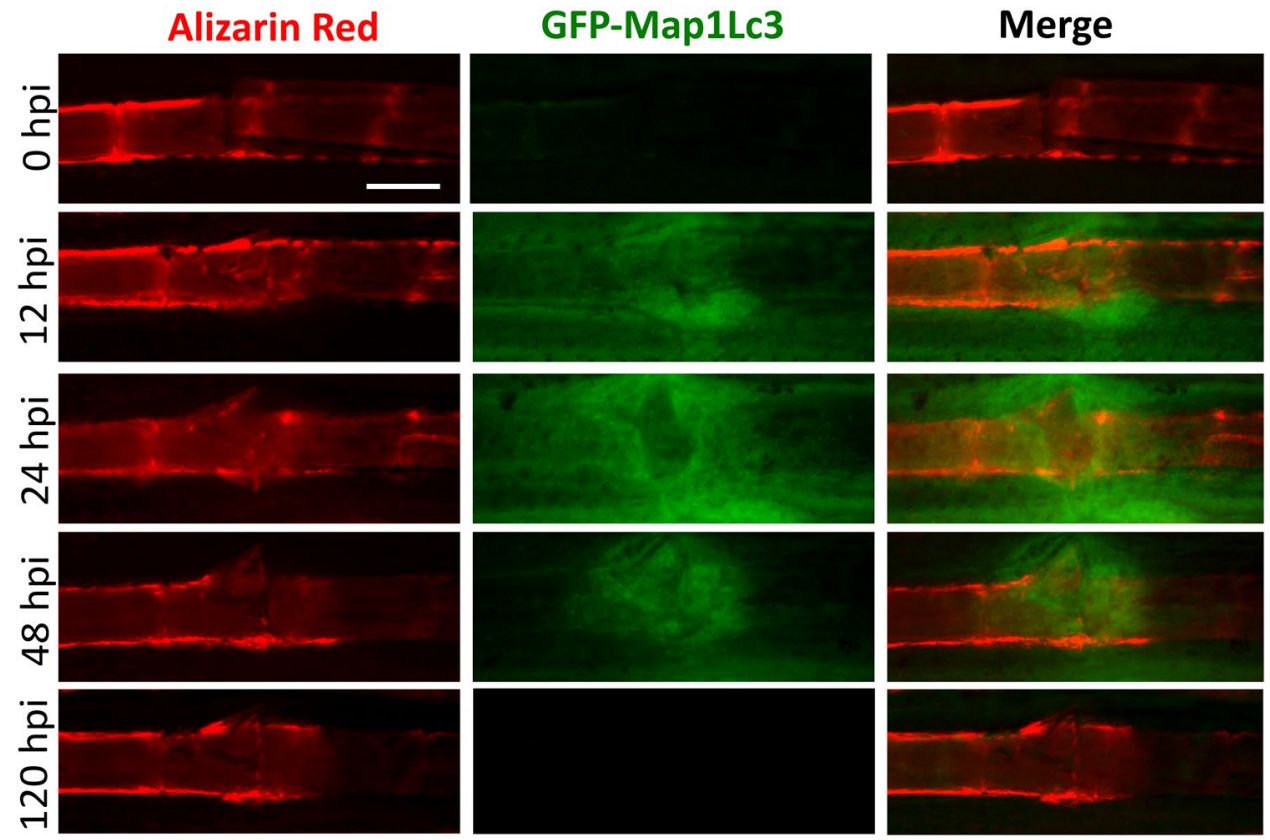

upregulated during the repair response and suggests a role for autophagy in bone repair. Similarly, these tools could also be utilised within the zebrafish scale system which was recently used to model the fracture repair response (Kobayashi-Sun et al. 2020). Using both of these models, the impact of autophagy modulating drugs, such as rapamycin, on the rate of fracture repair could be explored. Given that a number of bone disorders result in the development of weakened and fracture-prone bones, this could be particularly useful model for discovering potential bone repair therapies.

\section{Future directions and conclusion}

Existing studies have established zebrafish as a powerful model for studying vertebrate development and modelling genetic diseases, including various skeletal disorders (Carnovali et al. 2019; Kwon et al. 2019). Use of zebrafish in autophagy studies is more recent but growing nonetheless, and draws on the ease and plasticity of live imaging options they present, which simply cannot be paralleled in the more commonly used rodent autophagy models. As 
outlined above, initial studies using zebrafish autophagy models have already provided a new insight into the role of autophagy in skeletal cell differentiation and functioning, bone repair and regeneration, and drug discovery.

As these tools are further refined, the advantages of this model can be further exploited for autophagy research. For example, the development of conditional knockout lines of autophagy related genes for skeletal cells would enable the role of autophagy in these cells to be explored more accurately. Moreover, the development of inducible knockout lines would be especially helpful as the loss of essential autophagy proteins has proved to be developmentally lethal in several models (Dong et al. 2020; Mawed et al. 2019).

The use of cultured zebrafish scales as an ex vivo model is a very recent development that shows great potential for exploring the dynamics between autophagy and bone cell activity and function. As a flat but 3D whole skeletal preparation, autophagy assays similar to those done within in vitro models could be performed in zebrafish scales. This would help deepen our understanding of autophagy activity within osteoblasts and osteoclasts, and how different drugs or genetic mutations impact upon autophagy flux. The potential use of cultured scales in high throughput drug screening is also still yet to be fully explored. Together with zebrafish larvae, the scale system could help bridge the gap between modelling the complex biology of bones and rapidly testing and validating potential drugs.

As the tools available for genetic manipulation and in vivo imaging in zebrafish continue to improve, this model will be fundamental to progressing our understanding of autophagy cell biology and the significance of its role in skeletal development and pathology. Whilst it should be appreciated that not all observations from zebrafish, as with any animal model, are translatable to the human condition, zebrafish can still make a valuable contribution towards understanding the interactions between the autophagy pathway and the skeletal system, and in developing new therapeutics.

Acknowledgements We would like to thank the Wolfson Bioimaging Facility for confocal microscope (Leica SP5-II) access and imaging support. JM is supported by Wellcome Trust Dynamic Cell PhD programme 083474 and $\mathrm{CH}$ is supported by Versus Arthritis Senior Research Fellowship 21937.

Open Access This article is licensed under a Creative Commons Attribution 4.0 International License, which permits use, sharing, adaptation, distribution and reproduction in any medium or format, as long as you give appropriate credit to the original author(s) and the source, provide a link to the Creative Commons licence, and indicate if changes were made. The images or other third party material in this article are included in the article's Creative Commons licence, unless indicated otherwise in a credit line to the material. If material is not included in the article's Creative Commons licence and your intended use is not permitted by statutory regulation or exceeds the permitted use, you will need to obtain permission directly from the copyright holder. To view a copy of this licence, visit http://creativecommons.org/licenses/by/4.0/.

\section{References}

Aghajanian P, Mohan S (2018) The art of building bone: emerging role of chondrocyte-to-osteoblast transdifferentiation in endochondral ossification. Bone Res 6:19. https://doi.org/10.1038/ s41413-018-0021-z

Askary A et al (2016) Ancient origin of lubricated joints in bony vertebrates. Elife 5:e16415

Azzam E, Helfrich M, Hocking L (2011) Paget's disease-causing mutations in Sequestosome-1 impair autophagic protein degradation. J Bone Miner Res 26:1081

Barranco C (2015) Activate autophagy to prevent cartilage degeneration? Nat Rev Rheumatol 11:127-128

Bedell VM et al (2012) In vivo genome editing using a high-efficiency TALEN system. Nature 491:114-118

Berendsen AD, Olsen BR (2015) Bone development. Bone 80:14-18

Bergen DJ, Kague E, Hammond CL (2019) Zebrafish as an emerging model for osteoporosis: a primary testing platform for screening new osteo-active compounds. Front Endocrinol 10:6

Besio R et al (2018) 4-PBA ameliorates cellular homeostasis in fibroblasts from osteogenesis imperfecta patients by enhancing autophagy and stimulating protein secretion. Biochim Biophys Acta Mol Basis Dis 1864:1642-1652

Bird NC, Mabee PM (2003) Developmental morphology of the axial skeleton of the zebrafish, Danio rerio (Ostariophysi: Cyprinidae). Dev Dyn 228:337-357

Blommaart EF, Luiken JJ, Blommaart PJ, van Woerkom GM, Meijer AJ (1995) Phosphorylation of ribosomal protein S6 is inhibitory for autophagy in isolated rat hepatocytes. J Biol Chem 270:2320-2326

Bo $T$ et al (2016) Characterization of a relatively malignant form of osteopetrosis caused by a novel mutation in the PLEKHM1 gene. J Bone Miner Res 31:1979-1987

Bouderlique T, Vuppalapati KK, Newton PT, Li L, Barenius B, Chagin AS (2016) Targeted deletion of Atg5 in chondrocytes promotes age-related osteoarthritis. Ann Rheum Dis 75:627-631

Boya P, Reggiori F, Codogno P (2013) Emerging regulation and functions of autophagy. Nat Cell Biol 15:713-720

Brunt L, Skinner R, Roddy K, Araujo N, Rayfield E, Hammond C (2016) Differential effects of altered patterns of movement and strain on joint cell behaviour and skeletal morphogenesis. Osteoarthritis Cartilage 24:1940-1950

Cadwell K, Debnath J (2018) Beyond self-eating: The control of nonautophagic functions and signaling pathways by autophagyrelated proteins. J Cell Biol 217:813-822

Carames B, Taniguchi N, Otsuki S, Blanco FJ, Lotz M (2010) Autophagy is a protective mechanism in normal cartilage, and its aging-related loss is linked with cell death and osteoarthritis. Arthritis Rheum 62:791-801. https://doi.org/10.1002/ art. 27305

Caramés B, Taniguchi N, Otsuki S, Blanco FJ, Lotz M (2010) Autophagy is a protective mechanism in normal cartilage, and its aging-related loss is linked with cell death and osteoarthritis. Arthritis Rheum 62:791-801

Carnovali M, Banfi G, Mariotti M (2019) Zebrafish models of human skeletal disorders: embryo and adult swimming together. BioMed Res Int 2019:1-13 
Carnovali M, Luzi L, Banfi G, Mariotti M (2016) Chronic hyperglycemia affects bone metabolism in adult zebrafish scale model. Endocrine 54:808-817

Cheng NT, Meng H, Ma LF, Zhang L, Yu HM, Wang ZZ, Guo A (2017) Role of autophagy in the progression of osteoarthritis: The autophagy inhibitor, 3-methyladenine, aggravates the severity of experimental osteoarthritis. Int J Mol Med 39:1224-1232. https://doi.org/10.3892/ijmm.2017.2934

Choi AM, Ryter SW, Levine B (2013) Autophagy in human health and disease. N Engl J Med 368:651-662. https://doi.org/10.1056/ NEJMra1205406

Chung Y-H et al (2012) Microtubule-associated protein light chain 3 regulates Cdc42-dependent actin ring formation in osteoclast. Int J Biochem Cell Biol 44:989-997

Cinque L et al (2015) FGF signalling regulates bone growth through autophagy. Nature 528:272-275

Crotwell PL, Mabee PM (2007) Gene expression patterns underlying proximal-distal skeletal segmentation in late-stage zebrafish, Danio rerio. Dev Dyn 236:3111-3128

Cui J, Sim TH-F, Gong Z, Shen H-M (2012) Generation of transgenic zebrafish with liver-specific expression of EGFP-Lc3: a new in vivo model for investigation of liver autophagy. Biochem Biophys Res Commun 422:268-273

Cully M (2019) Zebrafish earn their drug discovery stripes. Nat Rev Drug Discov 18(11):811-814

Dallas SL, Bonewald LF (2010) Dynamics of the transition from osteoblast to osteocyte. Ann N Y Acad Sci 1192:437

de Vrieze E, Zethof J, Schulte-Merker S, Flik G, Metz JR (2015) Identification of novel osteogenic compounds by an ex-vivo sp7: luciferase zebrafish scale assay. Bone 74:106-113

DeSelm CJ et al (2011) Autophagy proteins regulate the secretory component of osteoclastic bone resorption. Dev Cell 21:966-974

Deter RL, De Duve C (1967) Influence of glucagon, an inducer of cellular autophagy, on some physical properties of rat liver lysosomes. J Cell Biol 33:437-449

Dikic I, Elazar Z (2018) Mechanism and medical implications of mammalian autophagy. Nat Rev Mol Cell Biol 19:349-364. https:// doi.org/10.1038/s41580-018-0003-4

Dong $\mathrm{G}$ et al (2020) Beclin 1 deficiency causes hepatic cell apoptosis via endoplasmic reticulum stress in zebrafish larvae. FEBS Lett 594:1155-1165

Dvornikov AV et al (2019) Phenotyping an adult zebrafish lamp2 cardiomyopathy model identifies mTOR inhibition as a candidate therapy. J Mol Cell Cardiol 133:199-208

Early JJ, Cole KL, Williamson JM, Swire M, Kamadurai H, Muskavitch M, Lyons DA (2018) An automated high-resolution in vivo screen in zebrafish to identify chemical regulators of myelination. Elife 7:e35136

Gioia R et al (2012) Impaired osteoblastogenesis in a murine model of dominant osteogenesis imperfecta: a new target for osteogenesis imperfecta pharmacological therapy. Stem Cells 30:1465-1476

Gomes P, Fernandes M (2011) Rodent models in bone-related research: the relevance of calvarial defects in the assessment of bone regeneration strategies. Lab Anim 45:14-24

Hall B, Miyake T (1992) The membranous skeleton: the role of cell condensations in vertebrate skeletogenesis. Anat Embryol 186:107-124

Hayes AJ et al (2013) Spinal deformity in aged zebrafish is accompanied by degenerative changes to their vertebrae that resemble osteoarthritis. PLoS ONE 8:e75787

He C, Bartholomew CR, Zhou W, Klionsky DJ (2009) Assaying autophagic activity in transgenic GFP-Lc3 and GFP-Gabarap zebrafish embryos. Autophagy 5:520-526

Hu Z, Chen B, Zhao Q (2019) Hedgehog signaling regulates osteoblast differentiation in zebrafish larvae through modulation of autophagy. Biol Open. https://doi.org/10.1242/bio.040840
Jiang P, Mizushima N (2014) Autophagy and human diseases. Cell Res 24:69-79

Kabashi E, Brustein E, Champagne N, Drapeau P (2011) Zebrafish models for the functional genomics of neurogenetic disorders. Biochim Biophys Acta Mol Basis Dis 1812:335-345

Kabeya Y et al (2000) LC3, a mammalian homologue of yeast Apg8p, is localized in autophagosome membranes after processing. The EMBO journal 19:5720-5728

Kawai M, Mödder UI, Khosla S, Rosen CJ (2011) Emerging therapeutic opportunities for skeletal restoration. Nat Rev Drug Discovery 10:141-156

Khuansuwan S, Barnhill LM, Cheng S, Bronstein JM (2019) A novel transgenic zebrafish line allows for in vivo quantification of autophagic activity in neurons. Autophagy 15:1322-1332

Klionsky DJ (2012) Look people, "Atg" is an abbreviation for "autophagy-related" That's it. Autophagy 8:1281

Klionsky DJ et al (2016) Guidelines for the use and interpretation of assays for monitoring autophagy. Autophagy 12:1-222

Kobayashi-Sun J et al (2020) Uptake of osteoblast-derived extracellular vesicles promotes the differentiation of osteoclasts in the zebrafish scale. Commun Biol 3:1-12

Kuma A, Komatsu M, Mizushima N (2017) Autophagy-monitoring and autophagy-deficient mice. Autophagy 13:1619-1628. https ://doi.org/10.1080/15548627.2017.1343770

Kwon RY, Watson CJ, Karasik D (2019) Using zebrafish to study skeletal genomics. Bone 126:37-50

Lamb CA, Yoshimori T, Tooze SA (2013) The autophagosome: origins unknown, biogenesis complex. Nat Rev Mol Cell Biol 14:759-774

Lawrence EA, Kague E, Aggleton JA, Harniman RL, Roddy KA, Hammond CL (2018) The mechanical impact of col11a2 loss on joints; col11a2 mutant zebrafish show changes to joint development and function, which leads to early-onset osteoarthritis. Phil Trans R Soc B 373:20170335

Lee W-S, Yoo W-H, Chae H-J (2015) ER stress and autophagy. Curr Mol Med 15:735-745

Levine B, Kroemer G (2019) Biological functions of autophagy genes: a disease perspective. Cell 176:11-42

Li D, Yu J, Xiao L, Miao W, Ji K, Wang S, Geng Y (2017) Autophagy attenuates the oxidative stress-induced apoptosis of Mc3T3-E1 osteoblasts. Eur Rev Med Pharmacol Sci 21:5548-5556

$\mathrm{Li} \mathrm{H}$ et al (2018) Defective autophagy in osteoblasts induces endoplasmic reticulum stress and causes remarkable bone loss. Autophagy 14:1726-1741

Lieberman AP, Puertollano R, Raben N, Slaugenhaupt S, Walkley SU, Ballabio A (2012) Autophagy in lysosomal storage disorders. Autophagy 8:719-730

Liu F et al (2013) Suppression of autophagy by FIP200 deletion leads to osteopenia in mice through the inhibition of osteoblast terminal differentiation. J Bone Miner Res 28:2414-2430

Liu K, Petree C, Requena T, Varshney P, Varshney GK (2019) Expanding the CRISPR toolbox in zebrafish for studying development and disease. Front Cell Dev Biol 7:13

Lu DL et al (2019) Fasting enhances cold resistance in fish through stimulating lipid catabolism and autophagy. J Physiol 597:1585-1603

Ma Y et al (2018) Autophagy controls mesenchymal stem cell properties and senescence during bone aging. Aging Cell 17:e12709

Mackay EW, Apschner A, Schulte-Merker S (2013) A bone to pick with zebrafish. BoneKEy Rep. https://doi.org/10.1038/bonek ey.2013.179

Mathai BJ, Meijer AH, Simonsen A (2017) Studying autophagy in zebrafish. Cells. https://doi.org/10.3390/cells6030021

Mathias JR, Saxena MT, Mumm JS (2012) Advances in zebrafish chemical screening technologies. Future med Chem 4:1811-1822 
Mawed SA, Zhang J, Ren F, Mei J (2019) Autophagy-related genes atg7 and beclin1 are essential for energy metabolism and survival during the larval-to-juvenile transition stage of zebrafish. BioRxiv 9(2):e1003279

Meneghetti G et al (2019) The epg5 knockout zebrafish line: a model to study Vici syndrome. Autophagy 15:1438-1454

Mizushima N (2007) Autophagy: process and function. Genes Dev 21:2861-2873. https://doi.org/10.1101/gad.1599207

Mizushima N, Levine B (2010) Autophagy in mammalian development and differentiation. Nat Cell Biol 12:823-830

Mizushima N, Yamamoto A, Matsui M, Yoshimori T, Ohsumi Y (2004) In vivo analysis of autophagy in response to nutrient starvation using transgenic mice expressing a fluorescent autophagosome marker. Mol Biol Cell 15:1101-1111

Mortensen M et al (2011) The autophagy protein Atg7 is essential for hematopoietic stem cell maintenance. J Exp Med 208:455-467

Mostowy S et al (2013) The zebrafish as a new model for the in vivo study of Shigella flexneri interaction with phagocytes and bacterial autophagy. PLoS Pathog 9:e1003588

Mundlos S, Olsen B (1997) Heritable diseases of the skeleton. Part I: Molecular insights into skeletal development-transcription factors and signaling pathways. FASEB J 11:125-132

Nollet M et al (2014) Autophagy in osteoblasts is involved in mineralization and bone homeostasis. Autophagy 10:1965-1977. https://doi. org/10.4161/auto.36182

Nuschke A, Rodrigues M, Stolz DB, Chu CT, Griffith L, Wells A (2014) Human mesenchymal stem cells/multipotent stromal cells consume accumulated autophagosomes early in differentiation. Stem Cell Res Ther 5:140

Ochotny N, Voronov I, Owen C, Aubin JE, Manolson MF (2013) The R740 S mutation in the V-ATP ase a3 subunit results in osteoclast apoptosis and defective early-stage autophagy. J Cell Biochem 114:2823-2833

Oliver L, Hue E, Priault M, Vallette FM (2012) Basal autophagy decreased during the differentiation of human adult mesenchymal stem cells. Stem Cells Dev 21:2779-2788

Onal M et al (2013) Suppression of autophagy in osteocytes mimics skeletal aging. J Biol Chem 288:17432-17440

Papp D et al (2016) AUTEN-67, an autophagy-enhancing drug candidate with potent antiaging and neuroprotective effects. Autophagy 12:273-286

Park C-M et al (2016) Ginsenoside re inhibits osteoclast differentiation in mouse bone marrow-derived macrophages and zebrafish scale model. Mol Cells 39:855

Pasqualetti S, Banfi G, Mariotti M (2012) The zebrafish scale as model to study the bone mineralization process. J Mol Histol 43:589-595

Piemontese M, Onal M, Xiong J, Han L, Thostenson JD, Almeida M, O'Brien CA (2016) Low bone mass and changes in the osteocyte network in mice lacking autophagy in the osteoblast lineage. Sci Rep 6:1-13

Ralston SH (2013) Paget's disease of bone. N Engl J Med 368:644-650

Rennekamp AJ, Peterson RT (2015) 15 years of zebrafish chemical screening. Curr Opin Chem Biol 24:58-70

Riggs BL, Parfitt AM (2005) Drugs used to treat osteoporosis: the critical need for a uniform nomenclature based on their action on bone remodeling. J Bone Miner Res 20:177-184

Santos-Ledo A, Garcia-Macia M, Campbell PD, Gronska M, Marlow FL (2017) Kinesin-1 promotes chondrocyte maintenance during skeletal morphogenesis. PLoS Genet 13:e1006918

Sasaki T et al (2017) Autolysosome biogenesis and developmental senescence are regulated by both Spns1 and v-ATPase. Autophagy $13: 386-403$

Sasaki T et al (2014) Aberrant autolysosomal regulation is linked to the induction of embryonic senescence: differential roles of Beclin 1 and p53 in vertebrate Spns1 deficiency. PLoS Genet 10:e1004409. https://doi.org/10.1371/journal.pgen.1004409
Seda M et al (2019) An FDA-approved drug screen for compounds influencing craniofacial skeletal development and craniosynostosis. Mol Syndromol 10:98-114

Shen G et al (2018) Autophagy as a target for glucocorticoid-induced osteoporosis therapy. Cell Mol Life Sci 75:2683-2693

Shi J et al (2015) Glucocorticoids: Dose-related effects on osteoclast formation and function via reactive oxygen species and autophagy. Bone 79:222-232

Siddiqi FH et al (2019) Felodipine induces autophagy in mouse brains with pharmacokinetics amenable to repurposing. Nat Commun 10:1-14

Sommer NG, Hahn D, Okutan B, Marek R, Weinberg A-M (2019) Animal models in orthopedic research: the proper animal model to answer fundamental questions on bone healing depending on pathology and implant material. In: Animal models in medicine and biology. IntechOpen. https://doi.org/10.5772/intechopen.89137

Sousa S, Valerio F, Jacinto A (2012) A new zebrafish bone crush injury model. Biol Open 1:915-921

Srinivas V, Bohensky J, Shapiro IM (2009) Autophagy: a new phase in the maturation of growth plate chondrocytes is regulated by HIF, mTOR and AMP kinase. Cells Tissues Organs 189:88-92

Takeshige K, Baba M, Tsuboi S, Noda T, Ohsumi Y (1992) Autophagy in yeast demonstrated with proteinase-deficient mutants and conditions for its induction. J Cell Biol 119:301-311

Talbot JC, Amacher SL (2014) A streamlined CRISPR pipeline to reliably generate zebrafish frameshifting alleles. Zebrafish 11:583-585

Tomecka MJ, Ethiraj LP, Sánchez LM, Roehl HH, Carney TJ (2019) Clinical pathologies of bone fracture modelled in zebrafish. Dis Models Mech 12:dmm037630

Varga M et al (2014) Autophagy is required for zebrafish caudal fin regeneration. Cell Death Differ 21:547-556

Vuppalapati KK et al (2015) Targeted deletion of autophagy genes Atg5 or Atg7 in the chondrocytes promotes caspase-dependent cell death and leads to mild growth retardation. J Bone Miner Res 30:2249-2261

Walker SL et al (2012) Automated reporter quantification in vivo: highthroughput screening method for reporter-based assays in zebrafish. PLoS ONE 7:e29916

Wang B, Abraham N, Gao G, Yang Q (2016) Dysregulation of autophagy and mitochondrial function in Parkinson's disease. Transl Neurodegener 5:19

Wang K, Niu J, Kim H, Kolattukudy PE (2011) Osteoclast precursor differentiation by MCPIP via oxidative stress, endoplasmic reticulum stress, and autophagy. J Mol Cell Biol 3:360-368

Watson CJ, Kwon RY (2015) Osteogenic programs during zebrafish fin regeneration. BoneKEy Rep. https://doi.org/10.1038/bonek ey.2015.114

Wei Y, Liu M, Li X, Liu J, Li H (2018) Origin of the autophagosome membrane in mammals. BioMed Res Int 2018:1-9

Wirawan E, Berghe TV, Lippens S, Agostinis P, Vandenabeele P (2012) Autophagy: for better or for worse. Cell Res 22:43-61

Xia X et al (2010) Glucocorticoid-induced autophagy in osteocytes. J Bone Miner Res 25:2479-2488

Zahm AM, Bohensky J, Adams CS, Shapiro IM, Srinivas V (2011) Bone cell autophagy is regulated by environmental factors. Cells Tissues Organs 194:274-278

Zhang L et al (2010) Pathway-based genome-wide association analysis identified the importance of regulation-of-autophagy pathway for ultradistal radius BMD. J Bone Miner Res 25:1572-1580

Zhang M et al (2013) Enhancement of chondrocyte autophagy is an early response in the degenerative cartilage of the temporomandibular joint to biomechanical dental stimulation. Apoptosis 18:423-434

Zhang R, Varela M, Forn-Cuni G, Torraca V, van der Vaart M, Meijer AH (2020) Deficiency in the autophagy modulator Dram1 exacerbates pyroptotic cell death of Mycobacteria-infected macrophages. Cell Death Dis 11:1-16 
Zhang R, Varela M, Vallentgoed W, Forn-Cuni G, van der Vaart M, Meijer AH (2019) The selective autophagy receptors Optineurin and p62 are both required for zebrafish host resistance to mycobacterial infection. PLoS Pathog 15:e1007329. https://doi.org/10.1371/journ al.ppat.1007329

Zhao Q, Shao J, Chen W, Li Y-P (2007) Osteoclast differentiation and gene regulation. Front Biosci 12:2519-2529
Zhao Y et al (2012) Autophagy regulates hypoxia-induced osteoclastogenesis through the HIF-1 $\alpha /$ BNIP3 signaling pathway. J Cell Physiol 227:639-648

Publisher's Note Springer Nature remains neutral with regard to jurisdictional claims in published maps and institutional affiliations. 